\title{
Fully resonant scalars on asymptotically AdS wormholes
}

\author{
Andrés Anabalon, ${ }^{1}$ Julio Oliva, ${ }^{2}$ and Constanza Quijada ${ }^{2}$ \\ ${ }^{1}$ Universidad Adolfo Ibañez, Dep. de Ciencias, Facultad de Artes Liberales, Viña del Mar 2520000, Chile \\ ${ }^{2}$ Departamento de Física, Universidad de Concepción, Casilla 160-C, Concepción 4030000, Chile
}

(Received 13 March 2019; published 13 May 2019)

\begin{abstract}
In this work, we show the existence of asymptotically anti-de Sitter (AdS) wormhole geometries in which the scalar probe has an equispaced, fully resonant spectrum, as that of a scalar on AdS spacetime, and explore its dynamics when nonlinearities are included. The spacetime is a solution of Einstein-Gauss-Bonnet theory with a single maximally symmetric vacuum. Introducing a nonminimal coupling between the scalar probe and the Ricci scalar remarkably leads to a fully resonant spectrum for a scalar field fulfilling reflective boundary conditions at both infinities. Applying perturbative methods, which are particularly useful for unveiling the dynamics at time scales of order $\varepsilon^{-2}$ (where $\varepsilon$ characterizes the amplitude of the initial perturbation), we observe both direct and inverse energy cascades between modes. This motivates us to explore the energy returns in the case in which the dynamics is dominated by a single mode. We find numerical and perturbative evidence that near-exact returns do exist in this regime. We also provide some comments on the fully backreracting case and provide a proof of the universality of the weakly nonlinear dynamics around AdS, in the context of Lovelock theories with generic couplings, up to times of order $\varepsilon^{-2}$.
\end{abstract}

DOI: $10.1103 /$ PhysRevD.99.104022

\section{INTRODUCTION}

General Relativity (GR) in dimensions higher than 4 can be extended, still fulfilling the requirements of second-order field equations and diffeomorphism invariance. In general, in dimensions $D \geq 5$, the precise combination of higher curvature terms can be added to the Einstein-Hilbert action, leading also to second-order field equations [1]. These combinations are dimensional continuations of the Euler densities of the lower, even dimensions. These combinations may appear as low-energy effective actions in string theory, as it is the case for the $\mathcal{R}^{2}$ term in the heterotic and bosonic string theories [2]. The simplest deformation from GR is obtained in five dimensions, in which the Einstein-GaussBonnet theory has the following action principle:

$$
\begin{aligned}
I\left[g_{\mu \nu}\right]= & \frac{1}{16 \pi G_{5}} \int\left[R-2 \Lambda+\alpha\left(R^{2}-4 R_{\mu \nu} R^{\mu \nu}+R_{\mu \nu \gamma \sigma} R^{\mu \nu \gamma \sigma}\right)\right] \\
& \times \sqrt{-g} d^{5} x
\end{aligned}
$$

Note that the coupling $\alpha$ has mass dimension -2 , and here it represents a free coupling.

For generic values of the couplings, the theory admits a local Lorentz invariance that can be made manifest in the

Published by the American Physical Society under the terms of the Creative Commons Attribution 4.0 International license. Further distribution of this work must maintain attribution to the author(s) and the published article's title, journal citation, and DOI. Funded by SCOAP ${ }^{3}$. first-order formulation in which the vielbein and the spin connection transform as a vector and connection of $S O(4,1)$, respectively. When $\alpha \Lambda=-3 / 4$, the local symmetry group is enlarged to $S O(4,2)$ [3], the theory admits a unique maximally symmetric anti-de Sitter (AdS) solution, and it has the maximum number of propagating degrees of freedom [4]. At this particular point, the space of solutions is also enlarged and contains, in addition to black holes [5] and analytic rotating solutions [6,7], asymptotically locally AdS wormholes [8]. The line element of the wormhole metric reads

$d s^{2}=\ell^{2}\left[-\cosh ^{2} \rho d t^{2}+d \rho^{2}+\cosh ^{2} \rho\left(d \varphi^{2}+d \Sigma_{2}^{2}\right)\right]$,

where $-\infty<t<+\infty,-\infty<\rho<+\infty, 0<\varphi \leq 2 \pi$, and $d \Sigma_{2}$ stands for the line element of a compact, smooth quotient of the pseudosphere with radius $3^{-1 / 2}$. Here, $\ell^{2}=4 \alpha=-3 / \Lambda$. The two asymptotically locally $\mathrm{AdS}_{5}$ regions $\rho \rightarrow \pm \infty$ are connected by a traversable throat located at $\rho=0$, and the spacetime is symmetric under the reflection $\rho \rightarrow-\rho$. This spacetime being devoid of singularities and horizons represents a soliton in the nonlinear Einstein-Gauss-Bonnet theory. ${ }^{1}$

\footnotetext{
${ }^{1}$ It is interesting to notice that a similar situation occurs for Einsteinian gravities [9], in which it was shown that a wormhole exists for a particular value of the coupling constants. See also Ref. [10] for Lorentzian wormholes in GR supported by a Skyrme field.
} 
The propagation of a scalar probe on the background geometry (2) was originally explored in Ref. [11], in which the focus was on the computation, in a closed form, of the normal frequencies fulfilling different possible boundary conditions. In Ref. [12], this problem was partially revisited, and it was shown that for a particular value of a nonminimal coupling with the scalar curvature the propagation of the scalar is controlled by an effective Schrödinger problem in a RosenMorse potential, for which the energies are proportional to the square of the frequencies of the scalar probe. Since the eigenvalues of the Schrödinger operator in Rosen-Morse potentials are quadratic in the mode number $n$ [13], the spectrum of the purely radial scalar probe turns out to be equispaced or fully resonant. Equispaced spectra play an important role in turbulent energy transfer leading to the nonperturbative AdS instability [14-19]. These kind of fully resonant spectra lead to a rich phenomenology that also appears in nonlinear models of different physical nature as in self-gravitating scalars on a spherical cavity in $3+1$ [20], on systems describing Bose-Einstein condensates [21], and vortex precession [22], as well as in the conformal dynamics on the Einstein universe [23]. A program to classify all the spacetimes in which a minimally coupled scalar probe may lead to an exactly solvable effective Schroedinger problem, suggestively dubbed "Klein-Gordonization," was initiated in Ref. [24].

In this paper, we go beyond the linear level and perturbatively explore different aspects of the nonlinear dynamics of a fully resonant, self-interacting scalar probe on the wormhole spacetime (2).

In Sec. II, we revisit with detail the linear propagation of a scalar probe with a precise nonminimal coupling with the Ricci scalar. The solutions to this linear problem are dubbed "wormhole oscillons," and we show that the spectrum of these oscillons is linear in the mode number. After introducing a self-interaction, in Sec. III, we construct the system of equations that control the dynamics of the infinite oscillators in the two-time framework (TTF) [25] or the time-averaged system [17-18]. This approach has been particularly useful in the context of the nonperturbative instability of AdS, since it captures the dynamics at times of order $\varepsilon^{-2}$, where $\varepsilon$ characterizes the energy content of the initial perturbation. By truncating the system of oscillators, we study the energy transfer between modes and show that there are direct and inverse energy cascades. In particular, when the dynamics is dominated by a single mode, we find evidence of near-exact energy returns, which is confirmed in Sec. IV analytically using perturbation theory. Section V is devoted to conclusions and further comments on the universality of the weakly nonlinear dynamics on AdS for Lovelock theories with generic couplings. It is well known that higher curvature gravity theories may have more than one maximally symmetric vacuum. We show that for an arbitrary Lovelock theory, provided the couplings are generic, the form of the equation for the infinite oscillators that control the dynamics in the TTF is universal.

\section{LINEAR SCALAR PROBE}

Let us consider the equation for a scalar probe on the wormhole geometry (2), with a fixed nonminimal coupling, ${ }^{2}$

$$
\left(\square-m^{2}-\frac{3}{8} R\right) \phi_{1}\left(x^{\mu}\right)=0 .
$$

We will see below that, even though the Ricci scalar is a nontrivial function of the radial coordinate

$$
R=-\frac{20}{\ell^{2}}+\frac{6}{\ell^{2} \cosh ^{2}(\rho)},
$$

the equation for the scalar probe can be solved analytically. Hereafter, for simplicity, we fix $\ell=1$.

Introducing a mode separation and considering only a radial spatial dependence

$$
\phi_{1}(t, \rho)=e^{-i \omega t} R(\rho)
$$

and the radial coordinate $\rho^{*}=2 \arctan \left(e^{\rho}\right)$ which maps $\rho \in] \infty, \infty\left[\right.$ to $\left.\rho^{*} \in\right] 0, \pi[$, we obtain

$$
-\frac{d^{2} S\left(\rho^{*}\right)}{d \rho^{* 2}}+U\left(\rho^{*}\right) S\left(\rho^{*}\right)=\omega^{2} S\left(\rho^{*}\right),
$$

where $S(\rho)=\cosh (\rho)^{3 / 2} R(\rho)$ and the effective potential reads

$$
U\left(\rho^{*}\right):=\frac{1}{4} \frac{4 m^{2}-15}{\sin ^{2}\left(\rho^{*}\right)}
$$

In terms of the coordinate $\rho^{*}$, the metric is manifestly conformal to the product of $\mathbb{R}_{t} \times \mathbb{R} \times S^{1} \times \Sigma_{2}$ and reads

$$
d s^{2}=\frac{1}{\sin ^{2}\left(\rho^{*}\right)}\left[-d t^{2}+d \rho^{* 2}+d \varphi^{2}+d \Sigma_{2}^{2}\right],
$$

and the wormhole boundaries are located at the divergences of the conformal factor.

Equation (6) is that of a quantum particle moving in a Rosen-Morse potential. For the following analysis, it is convenient to introduce the coordinate $z$ such that

$$
\tanh (\rho)=1-2 z
$$

which maps $\rho \in] \infty, \infty[$ to $z \in] 1,0[$. The wave equation (3) leads to

$$
\ddot{\phi_{1}}+L \phi_{1}=0
$$

\footnotetext{
${ }^{2}$ Note that this is not the conformal coupling since in general $\xi_{\text {conf }}=\frac{D-2}{4(D-1)}$.
} 
where the operator $L$ is defined as

$$
L:=-z^{2}(1-z)^{2} \frac{d}{d z}\left(\frac{1}{z(1-z)} \frac{d}{d z}\right)+\frac{m^{2}-15 / 2}{4 z(1-z)}+\frac{9}{4} .
$$

This operator admits the asymptotic behaviors

$$
\begin{aligned}
& R(z)^{z \rightarrow 0} D_{1} z^{\Delta_{+}}+D_{2} z^{\Delta_{-}}, \\
& R(z)^{z \rightarrow 1} \tilde{D}_{1}(1-z)^{\Delta_{+}}+\tilde{D}_{2}(1-z)^{\Delta_{-}},
\end{aligned}
$$

where $\Delta_{ \pm}=1 \pm \frac{1}{2} \sqrt{m^{2}-\frac{7}{2}}$. Note that $\Delta_{+}>0$, while $\Delta_{-} \leq 0$ for $m^{2} \geq 15 / 2$. Assuming $m^{2} \geq 15 / 2$ (which in global AdS would correspond to $m^{2} \geq 0$ ), we impose reflective boundary conditions at both boundaries $z=1$ and $z=0$, setting $D_{2}=\tilde{D}_{2}=0$, and consequently the operator $L$ is essentially self-adjoint on $L^{2}\left([1,0],-\frac{1}{z^{2}(1-z)^{2}}\right)$. This differential eigenvalue problem therefore leads to the following normal frequencies and normal modes:

$$
\begin{gathered}
\omega_{j}^{2}=\left(j+\frac{1}{2}+\sqrt{m^{2}-\frac{7}{2}}\right)^{2}, \\
e_{j}(z)=C_{j} z^{1+\frac{1}{2} \sqrt{m^{2}-\frac{7}{2}}}(1-z)^{1-\frac{1}{2} \sqrt{m^{2}-\frac{7}{2}}} \\
\times{ }_{2} F_{1}\left(-j-\sqrt{m^{2}-\frac{7}{2}}, 1+j+\sqrt{m^{2}-\frac{7}{2}} ; 1\right. \\
\left.+\sqrt{m^{2}-\frac{7}{2}} ; z\right) .
\end{gathered}
$$

The latter can also be written in terms of Jacobi polynomials $P_{n}^{(a, b)}(1-2 z)$, such that

$$
\begin{aligned}
e_{j}(z)= & D_{j} z^{1+\frac{1}{2} \sqrt{m^{2}-\frac{7}{2}}}(1-z)^{1-\frac{1}{2} \sqrt{m^{2}-\frac{7}{2}}} \\
& \times P_{j+\sqrt{m^{2}-\frac{7}{2}}}^{\left(\sqrt{m^{2}-\frac{7}{2}},-\sqrt{m^{2}-\frac{7}{2}}\right)}(1-2 z)
\end{aligned}
$$

where $C_{j}$ and $D_{j}$ are normalization constants that depend on the mass of the scalar probe and are proportional, the proportionality factor being a quotient of Gamma functions. Hereafter, we refer to (14) as wormhole oscillons. The general solution to the linear problem is given by an arbitrary superposition of the modes (14), leading to

$$
\phi_{1}(t, z)=\sum_{j=0}^{\infty} a_{j} \cos \left(\omega_{j} t+\beta_{j}\right) e_{j}(z) .
$$

For simplicity, and mimicking the massless case in AdS, we set $m^{2}=15 / 2$. In this case, the normalization constants in (14) fulfill
$2 C_{j}=[(j+1)(j+2)(j+3)(j+4)(5+2 j)]^{1 / 2}$,

leading to $\left(e_{i}(z), e_{j}(z)\right)=\delta_{i j}$. The frequencies which were already equispaced in (13) further reduce to

$$
\omega_{j}=\frac{5}{2}+j
$$

with $j=0,1,2, \ldots$.

Note that we have been able to find a fully resonant, equispaced spectrum for a scalar probe propagating on a spacetime with nontrivial topology. Below, we introduce a self-interaction on the scalar to characterize the energy transfer between modes. As mentioned above, it has been shown that such a problem captures some features of the backreacting, massless scalar in AdS (see, e.g., Refs. [26,27]).

\section{SELF-INTERACTING SCALAR PROBE}

Now, we will introduce a nonlinearity in the scalar probe we discussed in the previous section. In particular, we will focus on

$$
\square \phi-\left(m^{2}+\frac{3}{8} R\right) \phi-\frac{\lambda}{3 !} \phi^{3}=0,
$$

setting $m^{2}=15 / 2$, since this value leads to $\Delta_{-}=0$ in (12) and mimics the massless case in AdS. Here, $\lambda$ is a constant with mass dimension -1 . Following the TTF, we introduce the slow time $\tau=\epsilon^{2} t$ and the perturbative ansatz

$$
\phi(t, \tau, z)=\sum_{j=0}^{\infty} \epsilon^{2 j+1} \phi_{2 j+1}(t, \tau, z)
$$

Note that a direct perturbative approach leads to resonant terms, some of which could be perturbatively absorbed by a Poincare-Lindstedt shift. The TTF helps deal with this feature, and even more, its validity is ensured at least up to times of order $\epsilon^{-2}$. Naturally, at first order in $\epsilon$, one reobtains the linear problem

$$
\partial_{t}^{2} \phi_{1}+L \phi_{1}=0
$$

where the operator $L$ is given in (11), leading to

$$
\phi_{1}(t, \tau, x)=\sum_{l=0}^{\infty}\left(A_{l}(\tau) e^{-i \omega_{l} t}+\bar{A}_{l}(\tau) e^{i \omega_{l} t}\right) e_{l}(x),
$$

where $A_{l}(\tau)$ are arbitrary functions of the slow time $\tau$. Here, $\bar{A}_{l}(\tau)$ stands for the complex conjugate of $A_{l}(\tau)$. At the next perturbative order in $\epsilon$, one obtains

$$
\partial_{t}^{2} \phi_{3}+L \phi_{3}+2 \partial_{t} \partial_{\tau} \phi_{1}=S(t, \tau, x),
$$



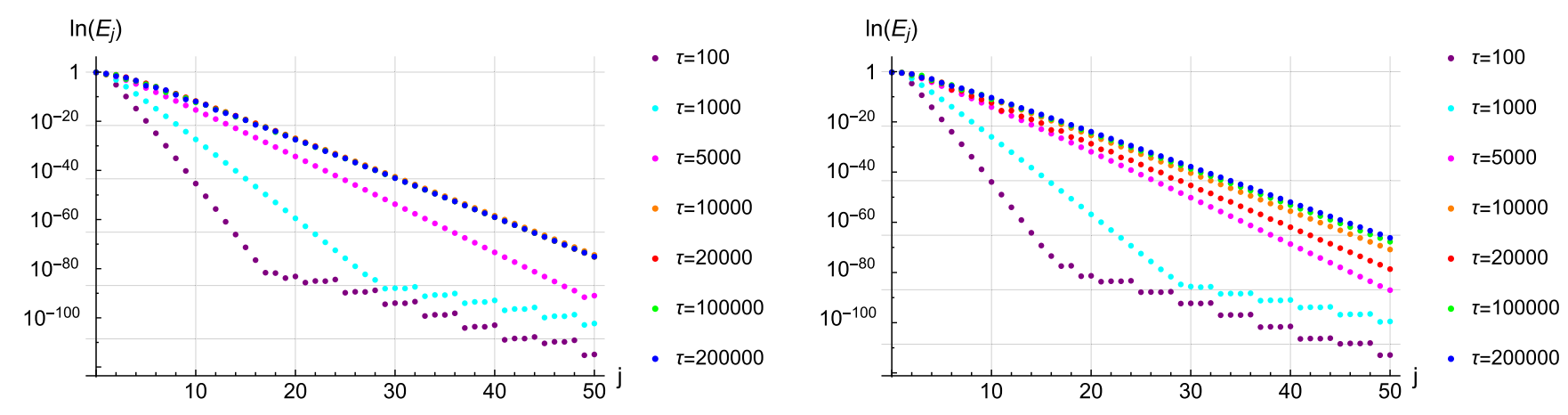

FIG. 1. Evolution of the energy per mode as a function of the mode number $j$, for $\left(E_{0}(0), E_{1}(0)\right)=(3 / 4,1 / 4)$ (left panel) and $\left(E_{0}(0), E_{1}(0)\right)=(1 / 2,1 / 2)$ (right panel). For late times, the energy per mode is exponentially suppressed for large $j$, i.e., $E_{j} \sim e^{-j}$.

with the source given by the lower-order term $S=-\frac{1}{24} \frac{\phi_{1}^{3}}{z(1-z)}$. Here, one proposes a solution for $\phi_{3}$ of the form

$$
\phi_{3}(t, \tau, x)=\sum_{n}^{\infty}\left(B_{n}(t, \tau)+\bar{B}_{n}(t, \tau)\right) e_{n}(x) .
$$

Projecting Eq. (23) on the basis of wormhole oscillons, one obtains

$$
\begin{aligned}
& \partial_{t}^{2}\left(B_{j}+\bar{B}_{j}\right)+\omega_{j}^{2}\left(B_{j}+\bar{B}_{j}\right)-2 i \omega_{j}\left(\partial_{\tau} A_{j} e^{-i \omega_{j} t}-\partial_{\tau} \bar{A}_{j} e^{i \omega_{j} t}\right) \\
& \quad=\left(e_{j}, S\right),
\end{aligned}
$$

with

$$
\begin{aligned}
\left(e_{j}, S\right)= & \sum_{n, l, m} \frac{\mathcal{S}_{j n l m}}{3}\left(A_{l} A_{m} A_{n} e^{-i\left(\omega_{l}+\omega_{m}+\omega_{n}\right) t}\right. \\
& +3 A_{l} A_{m} \bar{A}_{n} e^{-i\left(\omega_{l}+\omega_{m}-\omega_{n}\right) t} \\
& +3 A_{l} \bar{A}_{m} \bar{A}_{n} e^{-i\left(\omega_{l}-\omega_{m}-\omega_{n}\right) t} \\
& \left.+\bar{A}_{l} \bar{A}_{m} \bar{A}_{n} e^{-i\left(-\omega_{l}-\omega_{m}-\omega_{n}\right) t}\right),
\end{aligned}
$$

where the interaction integrals $\mathcal{S}_{\text {jnlm }} \in \mathbb{R}$ are defined as

$$
\mathcal{S}_{j n l m}=\frac{1}{8} \int_{1}^{0} \frac{e_{j}(z) e_{n}(z) e_{l}(z) e_{m}(z)}{z^{3}(1-z)^{3}} d z
$$

The TTF equations are obtained by imposing that the functions $A(\tau)$ that appear in the lhs of Eq. (25) exactly cancel the resonant terms coming from the rhs of the same equation. This leads to

$$
\begin{aligned}
-2 i \omega_{j} \partial_{\tau} A_{j}= & \sum_{n, l, m} \frac{\mathcal{S}_{j n l m}}{3}\left[A_{l} A_{m} A_{n} \delta_{\omega_{j}, \omega_{l}+\omega_{m}+\omega_{n}}\right. \\
& +3 A_{l} A_{m} \bar{A}_{n} \delta_{\omega_{j}, \omega_{l}+\omega_{m}-\omega_{n}} \\
& +3 A_{l} \bar{A}_{m} \bar{A}_{n} \delta_{\omega_{j}, \omega_{l}-\omega_{m}-\omega_{n}} \\
& \left.+\bar{A}_{l} \bar{A}_{m} \bar{A}_{n} \delta_{\omega_{j},-\omega_{l}-\omega_{m}-\omega_{n}}\right]
\end{aligned}
$$

We observe that the integrals $\mathcal{S}_{\text {jnlm }}$ are nonvanishing only in the channel $\omega_{j}+\omega_{n}=\omega_{l}+\omega_{m}$ (or equivalently $j+n=$ $l+m$ ), leading to the TTF equations

$$
-2 i \omega_{j} \partial_{\tau} A_{j}=\sum_{j+n=m+l} \mathcal{S}_{j n l m} A_{l} A_{m} \bar{A}_{n} .
$$

The vanishing of the channels $\omega_{j}=\omega_{l}+\omega_{m}+\omega_{n}$ and $\omega_{j}=\omega_{l}-\omega_{m}-\omega_{n}$ is a special property of our equispaced spectrum, as in AdS. The vanishing of the overlap integrals $\mathcal{S}$ in these cases can be proved using the expressions for the wormhole oscillons in terms of Jacobi polynomials in (15), as for a scalar probe in AdS [28-29]. Observing the symmetries in the indices of the overlap integrals $\mathcal{S}_{j n l m}$ and the fact they vanish unless $j+n=m+l$, one can deduce that the total energy $E=\sum_{j} \omega_{j}^{2} \bar{A}_{j}(\tau) A_{j}(\tau)$ and the "particle number" $N=\sum_{j} \omega_{j} \bar{A}_{j}(\tau) A_{j}(\tau)$ are conserved $[18,26,30]$. The conservation of these quantities is particularly useful for monitoring the stability of the numerical integration of the truncated version of the system (29).

In what follows, we will solve the system of oscillators by truncating the sum up to order $j=j_{\max }$, for different initial data. We monitor the convergence by increasing $j_{\max }$ and study the energy transfer between modes induced by the nonlinearities.

In Fig. 1, we plot the evolution of the spectrum, showing energy transfer induced by the nonlinearities, for different initial conditions. We have evolved the truncated TTF system with $j_{\max }=50$. The spectra stabilize after some time, showing an exponential suppression of the energy as a function of the mode number. As it occurs for nonbackreacting probes in AdS, these spectra suggest the absence of a turbulent phenomenology. ${ }^{3}$

Figure 2 shows the actual time evolution of the energy per mode, for different initial conditions with $j_{\max }=50$. Even though the energy is initially distributed only in the fundamental and first excited modes, the nonlinearities

\footnotetext{
${ }^{3}$ See, e.g., Ref. [31] for a turbulent characterization of the power spectrum in $D=4,5$ in GR.
} 

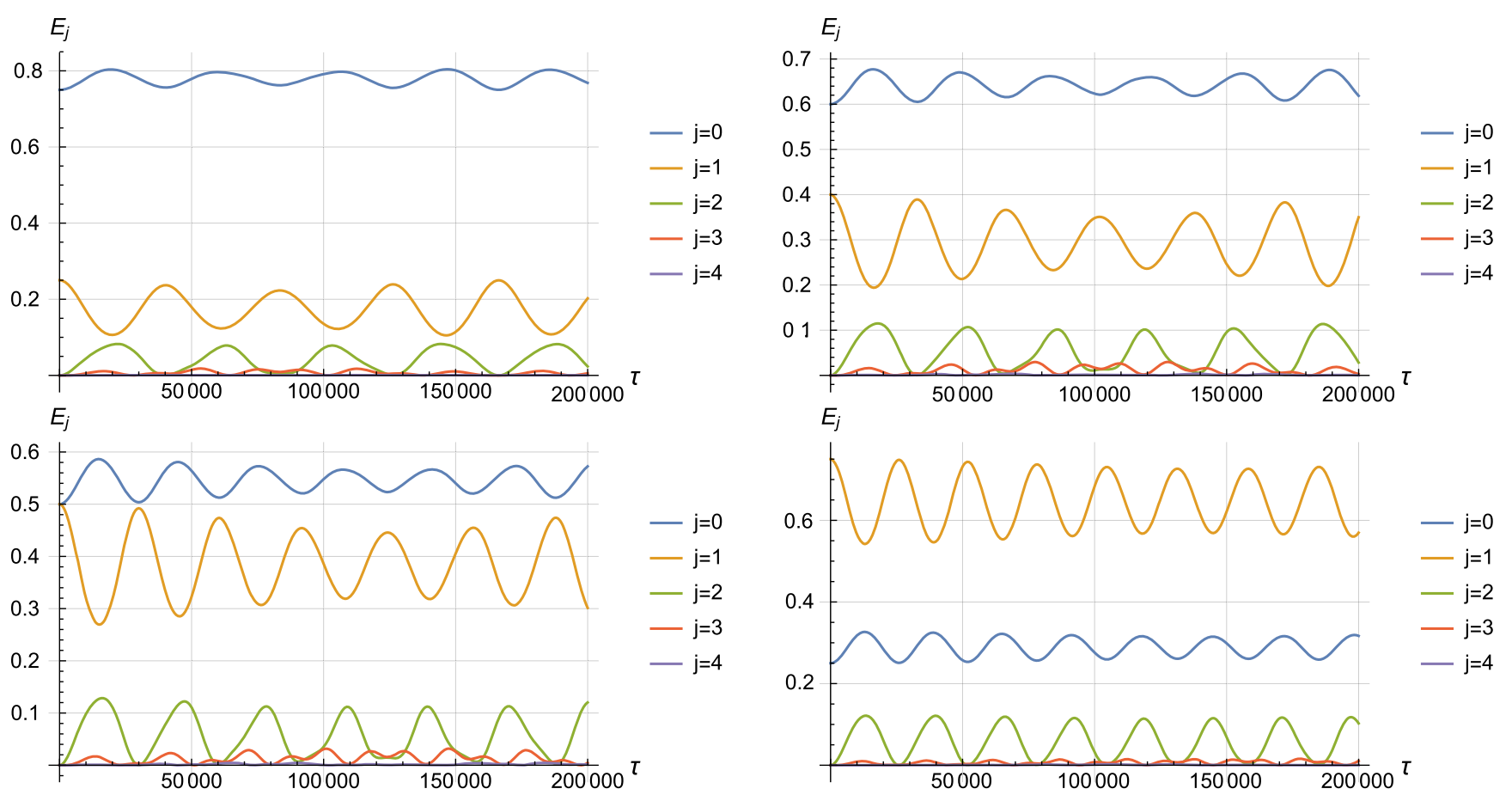

FIG. 2. The plots present the evolution of the energy per mode, for different initial excitations in the fundamental and first excited mode. The upper left panel corresponds to $\left(E_{0}(0), E_{1}(0)\right)=(3 / 4,1 / 4)$; for the upper right panel, we have used $\left(E_{0}(0), E_{1}(0)\right)=(3 / 5,2 / 5)$; the lower left panel corresponds to the two-mode equal energy initial date $\left(E_{0}(0), E_{1}(0)\right)=(1 / 2,1 / 2)$; and, finally, for the lower right panel, $\left(E_{0}(0), E_{1}(0)\right)=(1 / 4,3 / 4)$.

transfer energy to the higher harmonics. The plots suggest energy returns after a finite time. In the next section, we provide perturbative evidence of near-exact energy returns for situations such as that depicted in the upper left panel, in which the dynamics is clearly dominated by the fundamental mode.
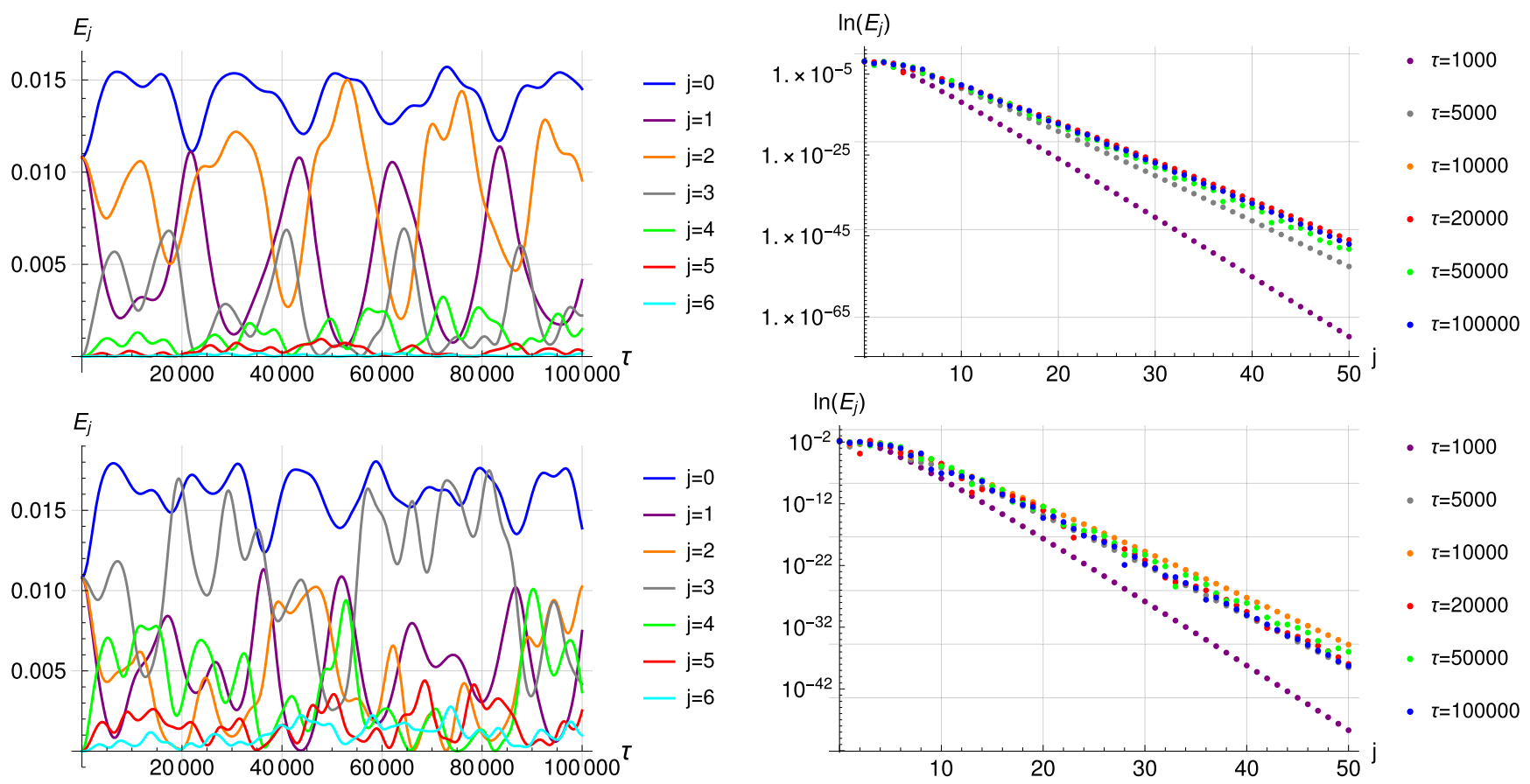

FIG. 3. Time evolution of the energy and spectra for three (upper panel) and four (lower panel) modes with equal energy as initial conditions. 

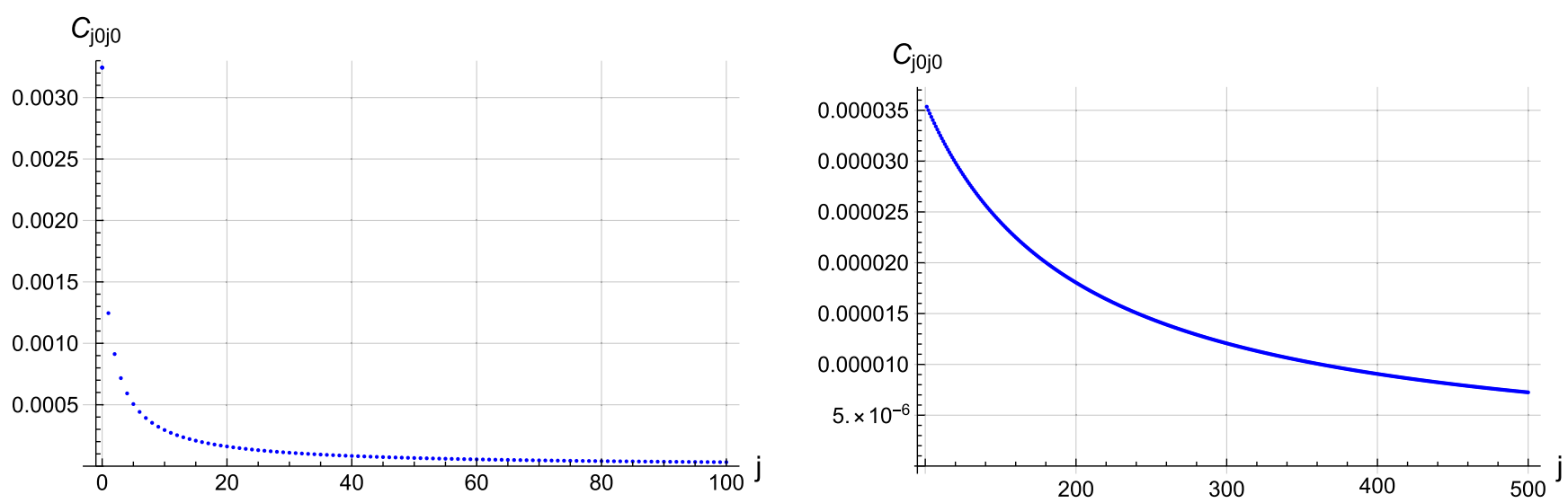

FIG. 4. The scaled overlap integral $C_{j 0 j 0}$ that determines the dynamics when the fundamental mode dominates.

It is also illustrative to consider initial data with three and four modes turned on. Figure 3 shows the time evolution of the energy content as well as the evolution of the stabilized spectra. Note that also in this case the energy in modes with large $j$ are exponentially suppressed.

\section{NEAR-EXACT ENERGY RETURNS}

Closely following Ref. [32], a perturbative argument can be given to have an analytic understanding of the (near-) exact energy returns suggested by Fig. 2. We will focus on the situation such that the dynamics is dominated by the fundamental mode as well as on the case in which the first excited mode dominates.

\section{A. Fundamental mode dominating the dynamics}

In particular, in the case in which the dynamics is dominated by the fundamental mode, we can introduce the scaled oscillators $q_{j}$ such that

$$
A_{j}(\tau)=\frac{q_{j}(\tau)}{\sqrt{\omega_{j}}} \delta^{j}
$$

with $\delta$ a small, perturbative parameter. The time-averaged system now reads

$$
i \partial_{\tau} q_{j}=\sum_{m=0}^{+\infty} \sum_{k=0}^{j+m} \delta^{2 m} C_{j, m, k, j+m-k} q_{k} q_{n+m-k} \bar{q}_{m}
$$

where

$$
C_{j, n, l, m}=-\frac{1}{2} \frac{\mathcal{S}_{j n l m}}{\sqrt{\omega_{j} \omega_{n} \omega_{l} \omega_{m}}}
$$

and the overlap integrals have been defined in (27). At leading order in $\delta$, we obtain the nonlinear system

$$
i \partial_{\tau} q_{j}=\bar{q}_{0} \sum_{k=0}^{j} C_{j, 0, k, j-k} q_{k} q_{n-k} .
$$

For $j=0$, the system leads to a decoupled, nonlinear equation for $q_{0}$, which is solved in a closed form, giving a constant modulus and a time-dependent phase for $q_{0} \in \mathbb{C}$. The global symmetries of the system can be used to set the absolute value of $q_{0}$ to 1 . Then, for $j \geq 1$, one obtains a set of linear equations that can be solved in a recursive manner, in which the $q_{k<j}$ 's appear as sources. The homogeneous equations depend only on the coefficients of the form $C_{j 0 j 0}$ (Fig. 4 depicts these integrals up to $j \sim 500$ ). A further use of the symmetries of the system allows us to set

$$
q_{0}(\tau)=e^{-i C_{0000} \tau} \quad \text { and } \quad q_{1}(\tau)=e^{-2 i C_{1010} \tau} .
$$

With this in mind, one can compute the time periods of the energies in the higher modes by computing the periods $T_{j}$ of $E_{j} \sim q_{j} \bar{q}_{j}$. Using our overlap integrals, we obtain $T_{2}=24024 \pi$ and $T_{3}=17 T_{2}, T_{4}=19 T_{3}, T_{5}=T_{4}$, $T_{6}=23 T_{5}$. Note that the ratios of the frequencies are relatively simple fractions (a simple, pictorial method to see the exact and near-exact returns is outlined in Fig. 5). The commensurability of the periods of the energy ensure exact energy returns at finite time within this perturbative approach. Nevertheless, it must be noted that the periods $T_{j}$ are an increasing function of the mode number $j$, and therefore as more modes are included in the analysis, one should have to wait longer for observing the recurrence. Note that higher modes are suppressed as $\delta^{j}$.

\section{B. First excited mode dominating the dynamics}

The lower-right panel of Fig. 2 depicts a case in which the first excited mode is dominating the dynamics of the energy content in the system. We can analytically explore such a case in a perturbative manner by introducing the ansatz 


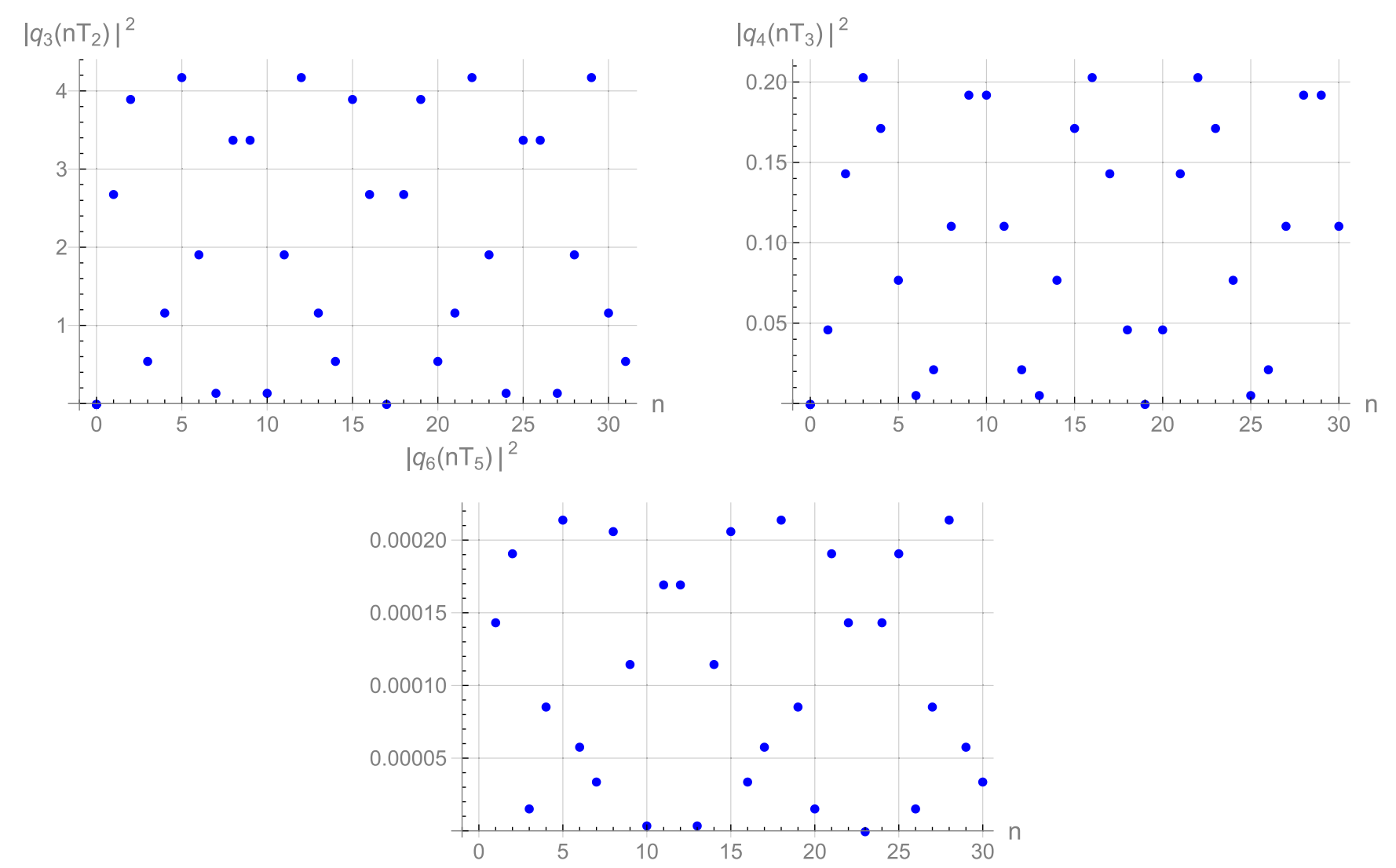

FIG. 5. The figures depict the exact and near-exact energy returns for the modes with $j=3$, 4, and 6 . Since in the initial condition these modes do not have energy, the exact returns are obtained once the dots intersect the horizontal line.

$$
A_{0}=\frac{q_{0}(\tau)}{\sqrt{\omega_{0}}} \delta \quad \text { and } \quad A_{j \geq 1}(\tau)=\frac{q_{j}(\tau)}{\sqrt{\omega_{j}}} \delta^{j-1}
$$

Retaining the leading contributions as $\delta$ goes to zero, one obtains

$$
i \dot{q}_{0}=C_{0211} \bar{q}_{2} q_{1}^{2}+2 C_{0101}\left|q_{1}\right|^{2} q_{0}
$$

and

$i \dot{q}_{n}=\bar{q}_{1} \sum_{k=1}^{n} C_{n, 1, k, n+1-k} q_{k} q_{n+1-k}+\bar{q}_{0} \sum_{k=1}^{n-1} C_{n, 0, k, n-k} q_{k} q_{n-k}$,

where the couplings $C$ 's have been defined in (32). As before, using the global symmetries, the time dependence of the leading oscillator appears in its phase, while the time dependence of the first two subleading oscillators leads to equal periods of their energy content given by $T_{0}=T_{2}=14586 \sqrt{14 / 635} \pi$. For the subsubleading modes, after some manipulation (see Sec. 4.2 of Ref. [32]), one obtains that $q_{3}(\tau)$ is a superposition of noncommesurable oscillations (see Fig. 6).

It is important to notice that in both of the cases developed in Secs. IV. A and IV. B, the returns are only near exact due to the energy transfer to modes with higher values of $j$. Nevertheless, if one truncates the system to include only low modes, Secs. IV. A and IV. B differ. In the former, since the frequencies are commensurable, there will be near-exact as well as exact returns (of the truncated version), while in the latter, the noncommensurability of the

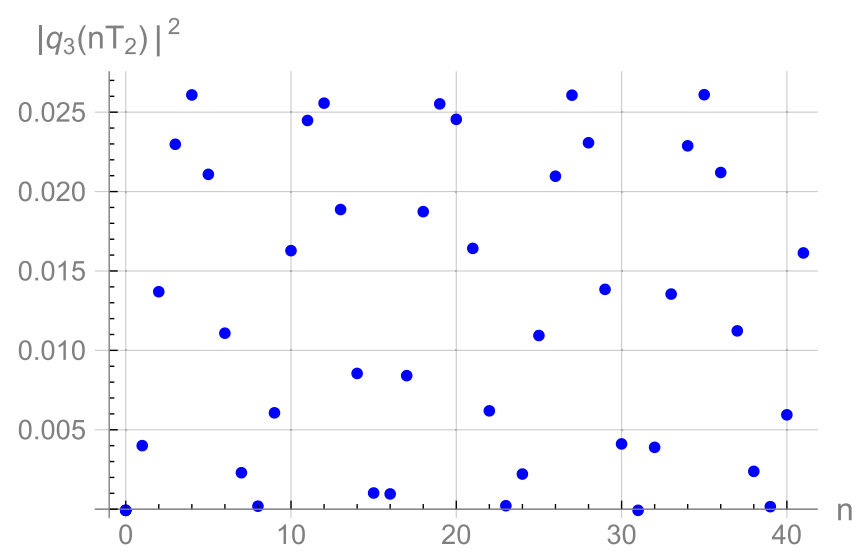

FIG. 6. This plot exhibits the near-exact returns of the energy in the third mode. Even though some points seem to lie on the horizontal axis, the actually do not touch it since, for example, for $n=31,\left|q_{3}\left(n T_{2}\right)\right|$ is of the order $10^{-7}$. 
modes will only allow for near-exact returns with different levels of precision, even in this truncated version.

\section{CONCLUSIONS}

In this work, we have shown the existence of a spacetime with nontrivial topology on which the linear dynamics of a scalar probe turn out to be fully resonant, leading to a rich phenomenology when nonlinearities are included. The fivedimensional wormhole geometry explored in this work can be generalized to arbitrary odd dimensions $D=2 n+1$ (with $n \geq 2$ )

$$
d s^{2}=\ell^{2}\left[-\cosh ^{2} \rho d t^{2}+d \rho^{2}+\cosh ^{2} \rho d \Sigma_{D-2}^{2}\right],
$$

as a solutions of Lovelock theory in the Chern-Simons case [3], provided the manifold $d \Sigma_{D-2}^{2}$ fulfills a suitable scalar constraint $[8,33,34,35]$. It was shown in Ref. [11] that a linear, nonminimally coupled scalar probe, fulfilling reflective boundary conditions has the spectrum

$$
\begin{aligned}
\omega_{n}^{2}= & \left(n+\frac{1}{2}+\sqrt{\left(\frac{D-1}{2}\right)^{2}+m_{\mathrm{eff}}^{2} \ell^{2}}\right)^{2} \\
& -\left(\frac{D-2}{2}\right)^{2}+Q+\xi[(D-1)(D-2)+\tilde{R}],
\end{aligned}
$$

where $m_{\mathrm{eff}}^{2}:=m^{2} \ell^{2}-D(D-1) \xi, \tilde{R}$ is the Ricci scalar of the Euclidean manifold $\Sigma_{D-2}$ (which we assume constant), and $Q$ stands for an eigenvalue of the Laplace operator on such a Euclidean manifold, normalized as $\nabla_{\Sigma}^{2} Y=-Q Y(Q$ being positive if $\Sigma$ is compact and without boundary). Generically, this spectrum will be only asymptotically resonant; nevertheless, in the particular case in which

$$
\left(\frac{D-2}{2}\right)^{2}-Q-\xi[(D-1)(D-2)+\tilde{R}]=0,
$$

the spectrum will be exactly equispaced. For a given rotational dependence of the scalar probe, i.e., for a fixed value of $Q$, resonance can be achieved for a particular value of the nonminimal coupling parameter $\xi$, providing a new, infinite family of gravitational backgrounds with fully resonant, equispaced spectrum for scalars probes.

\section{A. Universality of the weakly nonlinear dynamics in Lovelock theories}

Some comments on the backreacting case are in order. For the Einstein-Gauss-Bonnet theory, the scalar field collapse in AdS has been explored [36]. As shown below, remarkably, one can give a general analysis of the perturbative TTF, time-averaged approach in a generic Lovelock theory, in AdS. The field equations of Lovelock theories coupled to a massless scalar (the analysis can be trivially extended to the massive case) read

$$
\mathcal{E}_{\mu \nu}:=\sum_{k=0}^{[D / 2]} \alpha_{k} E_{\mu \nu}^{(k)}-T_{\mu \nu}=0
$$

where $T_{\mu \nu}$ stands for the stress-energy tensor of the minimally coupled scalar field $\phi$ and the Lovelock tensor of order $k$ is defined as

$E_{\mu \nu}^{(k)}:=-\frac{1}{2^{k+1}} g_{(\mu \mid \sigma} \delta_{\mid \nu) \gamma_{1} \cdots \gamma_{2 k}}^{\sigma \rho_{1} \cdots \rho_{2 k}} R^{\gamma_{1} \gamma_{2}} \rho_{1} \rho_{2} \cdots R^{\gamma_{2 k-1} \gamma_{2 k}} \rho_{2 k-1} A_{2 k}$.

Here, the couplings $\alpha_{k}$ are dimensionful.

Consider a metric of the form

$d s_{n}^{2}=g_{\mu \nu} d x^{\mu} d x^{\nu}=g_{a b}^{(2)}\left(y^{c}\right) d y^{a} d y^{b}+F^{2}(y) d \Omega_{S^{n-2}}^{2}$,

where $d \Omega_{S^{n-2}}$ stands for the line element of the $(n-2)$ sphere; $g_{a b}^{(2)}$ is a metric on a two-dimensional, Lorentzian manifold $M_{2}$; and $F(y)$ is a scalar on $M_{2}$.

The components of the $p$ th Lovelock tensor along the two-dimensional manifold $M_{2}$ were explicitly computed in Ref. [37] and read

$$
\begin{aligned}
E_{a b}^{(k)}= & -\frac{k(n-2) !}{(n-2 k-1) !} \frac{D_{a} D_{b} F-g_{c d}^{(2)} D^{c} F D^{d} F g_{a b}^{(2)}}{F} \\
& \times\left(\frac{1-g_{e f}^{(2)} D^{e} F D^{f} F}{F^{2}}\right)^{k-1} \\
& -\frac{(n-2) !}{2(n-2 k-2) !} g_{a b}^{(2)}\left(\frac{1-g_{e f}^{(2)} D^{e} F D^{f} F}{F^{2}}\right)^{k},
\end{aligned}
$$

where $D_{a}$ is the Levi-Civita covariant derivative on $M_{2}$. As usual, due to diffeomorphism invariance and spherical symmetry, the Lovelock equations along the angles in $S^{n-2}$ are a consequence of the equation along $M_{2}$ and the equation for the scalar field. The expression for the Lovelock equations on the metric of our interest

$$
\begin{aligned}
d s^{2}= & \frac{L^{2}}{\cos ^{2}(x)}\left[-e^{-2 f(t, x)} A(t, x) d t^{2}\right. \\
& \left.+\frac{d x^{2}}{A(t, x)}+\sin ^{2}(x) d \Omega_{S^{n-2}}^{2}\right]
\end{aligned}
$$

can be directly obtained by setting $F\left(y^{a}\right)=\tan x$ and $g_{a b}^{(2)}$ as the metric along the $(t, x)$ directions in (44). Following Ref. [38], here, we will consider the scaled slow time

$$
\tau=s_{1} \varepsilon^{2} t
$$

where $s_{1}$ and $s_{2}$ (below) are finite constants to be fixed at convenience. We will consider the expansions

$$
A(t, \tau, x)=1+s_{2} \varepsilon^{2} A_{2}(t, \tau, x)+\mathcal{O}\left(\varepsilon^{4}\right),
$$




$$
\begin{gathered}
f(t, \tau, x)=s_{2} \varepsilon^{2} f_{2}(t, \tau, x)+\mathcal{O}\left(\varepsilon^{4}\right), \\
\phi(t, \tau, x)=\varepsilon \phi_{1}(t, \tau, x)+s_{2} \varepsilon^{3} \phi_{3}(t, \tau, x)+\mathcal{O}\left(\varepsilon^{5}\right) .
\end{gathered}
$$

At the lowest order, the Lovelock equations determine the AdS radius $L$ in terms of the couplings $\alpha_{p}$, through the equation

$$
P\left[L^{-2}\right]:=\sum_{p=0}^{[D / 2]} \frac{\alpha_{p}(-1)^{p}}{(n-2 p-1) !}\left(\frac{1}{L^{2}}\right)^{p}=0,
$$

which defines the polynomial $P[\xi]$. The equation for the scalar field, at the lowest order, determine $\phi_{1}(t, x)$ as an arbitrary superposition of $n$-dimensional AdS oscillons. At order $\varepsilon^{2}$, one obtains the expressions for the $\mathcal{E}_{t}^{t}, \mathcal{E}_{x}^{x}$, and $\mathcal{E}_{x}^{t}$ equations in (41):

$$
\begin{aligned}
& -\frac{(n-2) !}{2 L^{2}} s_{2} \frac{d P\left[L^{-2}\right]}{d \xi}\left(\frac{A_{2}^{\prime}}{\tan x}-\frac{\left(2 \cos ^{2} x-n+1\right)}{\sin ^{2} x} A_{2}\right) \\
& =-\frac{\cos ^{2} x}{2 L^{2}}\left(\dot{\phi}_{1}^{2}+\phi_{1}^{\prime 2}\right), \\
& \quad-\frac{(n-2) !}{2 L^{2}} s_{2} \frac{d P\left[L^{-2}\right]}{d \xi} \frac{\dot{A}_{2}}{\tan x}=-\frac{\cos ^{2} x}{L^{2}} \phi_{1}^{\prime} \dot{\phi}_{1}, \\
& -\frac{(n-2) !}{2 L^{2}} s_{2} \frac{d P\left[L^{-2}\right]}{d \xi}\left(\frac{A_{2}^{\prime}}{\tan x}-\frac{\left(2 \cos ^{2} x-n+1\right)}{\sin ^{2} x} A_{2}-\frac{2 f_{2}^{\prime}}{\tan x}\right) \\
& =\frac{\cos ^{2} x}{2 L^{2}}\left(\dot{\phi}_{1}^{2}+\phi_{1}^{\prime 2}\right),
\end{aligned}
$$

where' and - denote derivatives with respect to $x$ and $t$, respectively. No derivatives with respect to the slow time appear at this order. From these equations, one can solve $A_{2}(t, x)$ and $f_{2}(t, x)$ as in GR. Then, substituting this at the next nontrivial order in the Klein-Gordon equation, one obtains

$$
\begin{aligned}
& \ddot{\phi}_{3}+\mathcal{L}\left[\phi_{3}\right]+\frac{2 s_{1}}{s_{2}} \partial_{t} \partial_{\tau} \phi_{1} \\
& =\phi_{1}^{\prime} \dot{A}_{2}+\phi_{1}^{\prime} A_{2}^{\prime}-\dot{\phi}_{1} \dot{f}_{2}-\phi_{1}^{\prime} f_{2}^{\prime}+2\left(f_{2}-A_{2}\right) \mathcal{L}\left[\phi_{1}\right],
\end{aligned}
$$

where the action of the operator $\mathcal{L}\left[\phi_{i}\right]$ is defined as

$$
\mathcal{L}\left[\phi_{i}\right]:=-\frac{\partial^{2} \phi_{i}}{\partial x^{2}}-\frac{(n-2)}{\cos x \sin x} \frac{\partial \phi_{i}}{\partial x} .
$$

Therefore, as in Ref. [38] for the Einstein-Gauss-Bonnet case in five dimensions, but now in the whole family of Lovelock theories, setting

$$
s_{1}=s_{2}=\left(\frac{d P\left[L^{-2}\right]}{d \xi}\right)^{-1}
$$

we see that the equations for the TTF approach, for a generic Lovelock theory (50)-(53), take exactly the same functional form as the equations in GR, provided we are expanding about AdS with a curvature corresponding to a simple zero of the polynomial (49), for which $\frac{d P\left[L^{-2}\right]}{d \xi}$ is nonvanishing. It is interesting to see that this polynomial completely controls the perturbative dynamics in Lovelock theories. On the other hand, for the wormhole studied in this work, the asymptotic AdS curvature radius exactly cancels the derivative of the polynomial (this occurs for any Chern-Simons theory within the Lovelock family), and therefore the perturbative approach in the backreacting situation does not apply. This is why we have focused on the probe limit of the scalar. Notwithstanding, the equations presented above for Lovelock theories are a signal of the universality of the weakly nonlinear dynamics up to times of order $\varepsilon^{-2}$, captured by the TTF, for generic values of the couplings, when the higher curvature terms belong to the Lovelock family. This was shown in Ref. [38] (see also Ref. [39]) for the Einstein-Gauss-Bonnet case, and here we have shown that such results extend to the whole family of Lovelock theories for generic values of the couplings. These results extend also for the family of quasitopological gravities $[40,41,42,43]$, since the dynamics on the spherically symmetric dynamical scenarios with matter can be obtained from the same formulas (50)-(52), by including extra terms in the polynomial. All the mentioned theories admit Birkhoff's theorems; therefore, on a spherically symmetric scenario, the dynamics is completely driven by the scalar.

It is well known that for Lovelock theories containing a $k$ th-order term in dimension $n=2 k+1$ the maximally symmetric AdS vacuum is gapped with respect to the smallest black hole (see, e.g., Refs. [44,45]), as it occurs for the Bañados-Teitelboim-Zanelli black hole [46], a feature that is captured by the numerical evolutions (see, e.g., Refs. [47,48] for the $2+1$ case and Ref. [49] for $4+1$ dimensions). It is interesting to note that the structure of the TTF dynamics, at times $\varepsilon^{-2}$, being universal for Lovelock theories with generic couplings, does not capture this gap. ${ }^{4}$

Finally, it would be interesting to explore the dynamics of a scalar collapse to a black hole in other asymptotically AdS solitons with two ends, including backreaction. The recently constructed analytic wormhole solution of General Relativity with a negative cosmological constant [51] defines a perfect scenario to initiate such exploration, since the Einstein-Klein-Gordon system leads to a wellposed initial boundary value problem in asymptotically AdS spacetimes.

\footnotetext{
${ }^{4}$ In Ref. [50], it was noted that in a direct perturbative at order $\varepsilon^{4}$ the presence of a Gauss-Bonnet term cannot be scaled out.
} 


\section{ACKNOWLEDGMENTS}

We would like to thank F. Correa, O. Evnin, O. Fierro, S. Green, L. Lehner, and H. Maeda for enlightening comments. This work was partially funded by FONDECYT Grants No. 1150246 and No. 1170279.

[1] D. Lovelock, J. Math. Phys. (N.Y.) 12, 498 (1971).

[2] B. Zwiebach, Phys. Lett. 156B, 315 (1985).

[3] M. Hassaine and J. Zanelli, Chern-Simons (Super)Gravity (100 Years of General Relativity) (World Scientific, Singapore, 2016).

[4] R. Troncoso and J. Zanelli, Classical Quantum Gravity 17, 4451 (2000).

[5] M. Banados, C. Teitelboim, and J. Zanelli, Phys. Rev. D 49, 975 (1994).

[6] A. Anabalon, N. Deruelle, Y. Morisawa, J. Oliva, M. Sasaki, D. Tempo, and R. Troncoso, Classical Quantum Gravity 26, 065002 (2009).

[7] A. Anabalon, N. Deruelle, D. Tempo, and R. Troncoso, Int. J. Mod. Phys. D 20, 639 (2011).

[8] G. Dotti, J. Oliva, and R. Troncoso, Phys. Rev. D 75, 024002 (2007).

[9] P. Bueno, P. A. Cano, R. A. Hennigar, and R. B. Mann, J. High Energy Phys. 10 (2018) 095.

[10] E. Ayon-Beato, F. Canfora, and J. Zanelli, Phys. Lett. B 752, 201 (2016).

[11] D. H. Correa, J. Oliva, and R. Troncoso, J. High Energy Phys. 08 (2008) 081.

[12] O. Fierro, D. Narbona, J. Oliva, C. Quijada, and G. Rubilar, arXiv:1812.02089.

[13] F. Cooper, A. Khare, and U. Sukhatme, Phys. Rep. 251, 267 (1995).

[14] P. Bizon and A. Rostworowski, Phys. Rev. Lett. 107, 031102 (2011).

[15] O. J. C. Dias, G. T. Horowitz, and J. E. Santos, Classical Quantum Gravity 29, 194002 (2012).

[16] V. Balasubramanian, A. Buchel, S. R. Green, L. Lehner, and S. L. Liebling, Phys. Rev. Lett. 113, 071601 (2014).

[17] B. Craps, O. Evnin, and J. Vanhoof, J. High Energy Phys. 10 (2014) 048.

[18] B. Craps, O. Evnin, and J. Vanhoof, J. High Energy Phys. 01 (2015) 108.

[19] P. Bizoń, M. Maliborski, and A. Rostworowski, Phys. Rev. Lett. 115, 081103 (2015).

[20] M. Maliborski, Phys. Rev. Lett. 109, 221101 (2012).

[21] A. Biasi, P. Bizon, B. Craps, and O. Evnin, Phys. Rev. E 98, 032222 (2018).

[22] A. Biasi, P. Bizon, B. Craps, and O. Evnin, Phys. Rev. A 96, 053615 (2017).

[23] P. Bizoń, B. Craps, O. Evnin, D. Hunik, V. Luyten, and M. Maliborski, Commun. Math. Phys. 353, 1179 (2017).

[24] O. Evnin, H. Demirchian, and A. Nersessian, Phys. Rev. D 97, 025014 (2018).

[25] V. Balasubramanian, A. Buchel, S. R. Green, L. Lehner, and S. L. Liebling, Phys. Rev. Lett. 113, 071601 (2014).
[26] P. Basu, C. Krishnan, and A. Saurabh, Int. J. Mod. Phys. A 30, 1550128 (2015).

[27] P. Basu, C. Krishnan, and P. N. Bala Subramanian, Phys. Lett. B 746, 261 (2015).

[28] I. S. Yang, Phys. Rev. D 91, 065011 (2015).

[29] O. Evnin and R. Nivesvivat, J. High Energy Phys. 01 (2016) 151.

[30] A. Buchel, S. R. Green, L. Lehner, and S. L. Liebling, Phys. Rev. D 91, 064026 (2015).

[31] H. P. de Oliveira, L. A. Pando Zayas, and E. L. Rodrigues, Phys. Rev. Lett. 111, 051101 (2013).

[32] A. Biasi, B. Craps, and O. Evnin, arXiv:1810.04753.

[33] G. Dotti, J. Oliva, and R. Troncoso, Phys. Rev. D 76, 064038 (2007).

[34] G. Dotti, J. Oliva, and R. Troncoso, Int. J. Mod. Phys. A 24, 1690 (2009).

[35] G. Dotti, J. Oliva, and R. Troncoso, Phys. Rev. D 82, 024002 (2010).

[36] N. Deppe, A. Kolly, A. R. Frey, and G. Kunstatter, J. High Energy Phys. 10 (2016) 087.

[37] H. Maeda, S. Willison, and S. Ray, Classical Quantum Gravity 28, 165005 (2011).

[38] A. Buchel, S. R. Green, L. Lehner, and S. L. Liebling, arXiv: 1410.5381.

[39] A. Buchel, Nucl. Phys. B896, 587 (2015).

[40] J. Oliva and S. Ray, Classical Quantum Gravity 27, 225002 (2010).

[41] R. C. Myers and B. Robinson, J. High Energy Phys. 08 (2010) 067.

[42] M. H. Dehghani, A. Bazrafshan, R. B. Mann, M. R. Mehdizadeh, M. Ghanaatian, and M. H. Vahidinia, Phys. Rev. D 85, 104009 (2012).

[43] A. Cisterna, L. Guajardo, M. Hassaine, and J. Oliva, J. High Energy Phys. 04 (2017) 066.

[44] C. Garraffo and G. Giribet, Mod. Phys. Lett. A 23, 1801 (2008).

[45] C. Charmousis, Lect. Notes Phys. 769, 299 (2009).

[46] M. Banados, C. Teitelboim, and J. Zanelli, Phys. Rev. Lett. 69, 1849 (1992).

[47] P. Bizoń and J. Jałmużna, Phys. Rev. Lett. 111, 041102 (2013).

[48] F. Pretorius and M. W. Choptuik, Phys. Rev. D 62, 124012 (2000).

[49] N. Deppe, C. D. Leonard, T. Taves, G. Kunstatter, and R. B. Mann, Phys. Rev. D 86, 104011 (2012).

[50] D. S. Menon and V. Suneeta, Phys. Rev. D 93, 024044 (2016).

[51] A. Anabalón and J. Oliva, arXiv:1811.03497. 